\title{
Parton Distribution Functions and Nuclear Effects
}

\author{
Alejandro Daleo* \\ Laboratorio de Física Teórica. Departamento de Física, Universidad Nacional de La Plata, C.C. \\ 67, 1900 La Plata, Argentina \\ E-mail: daleo@venus.fisica.unlp.edu.ar
}

ABSTRACT: We investigate the incidence of nuclear effects in the extraction of unpolarized parton densities by means of a global LO QCD fit including data produced using deuterium targets. The data considered include DIS, Drell-Yan and W-production asymmetry. We evaluate the consequences of nuclear corrections, through different models, in the analysis of the flavor symmetry breaking in the nucleon.

\section{Introduction}

Nowadays, the increasingly precision of hard lepton-hadron and hadron-hadron experimental data allows, not just to parameterize structure functions of nucleons but, to discriminate between quark and antiquark flavors in the extraction of parton distribution functions (PDFs) [1], permitting the test of features like flavor symmetry breaking at sea quark level, and other assumptions about the partonic structure of nucleons $[2,3,4]$.

Although nuclear effects are crucial in order to perform the flavor decomposition in the extraction of PDFs, in most global analysis only rather simple phenomenological models for them are considered, and in the case of deuteron target they are usually ignored, as they are small if not tiny. However, in some experiments they can be strongly amplified.

Indeed, in reference [5], it was proposed that nuclear effects in the deuteron give an alternative explanation for the Gottfried Sum Rule (GSR) violation [6]. There, it was shown how nuclear effects not only allowed to interpret NMC [7] data

${ }^{*}$ This work has been done in collaboration with C.A. García Canal (Laboratorio de Física Teórica. Departamento de Física, Universidad Nacional de La Plata, C.C. 67, 1900 La Plata, Argentina), G.A. Navarro and R. Sassot (Departamento de Física, Universidad de Buenos Aires, Ciudad Universitaria, Pab.1, 1428 Buenos Aires, Argentina). even preserving flavor symmetry, but also could bring accord between parton distributions available at that time and the experimental data.

Later, other experiments $[8,9]$ showed that indeed flavor symmetry breaking was there, however up to now the apparent discrepancy between NMC data (assuming no nuclear effects) and the prediction of the most recent sets of parton densities still persists.

The fact that $F_{2}^{p}, F_{2}^{d}$, and even $F_{2}^{n} / F_{2}^{p}$ data are reproduced with great accuracy by the fits (even neglecting nuclear effects), but at the same time the comparison with $\left(F_{2}^{p}-F_{2}^{n}\right)$ fails wretchedly, suggests the presence of some hitherto neglected effect.

In this contribution we explore the hypothesis of nuclear effects in the deuteron structure function being responsible for the discrepancy between NMC data and standard parton densities, and we evaluate consequences of this effects in the extraction of parton densities and in the study of flavour symmetry breaking within the nucleon sea quarks, implementing nuclear corrections in a global QCD fit including NMC data for $\left(F_{2}^{p}-F_{2}^{n}\right)$.

As result of this procedure, we find that the enforcement of the agreement with NMC data on $\left(F_{2}^{p}-F_{2}^{n}\right)$ without taking into account nuclear effects implies $\bar{d}-\bar{u}$ densities which fail to reproduce E866 Drell-Yan experiment data. A very simple model for moderate nuclear effects 

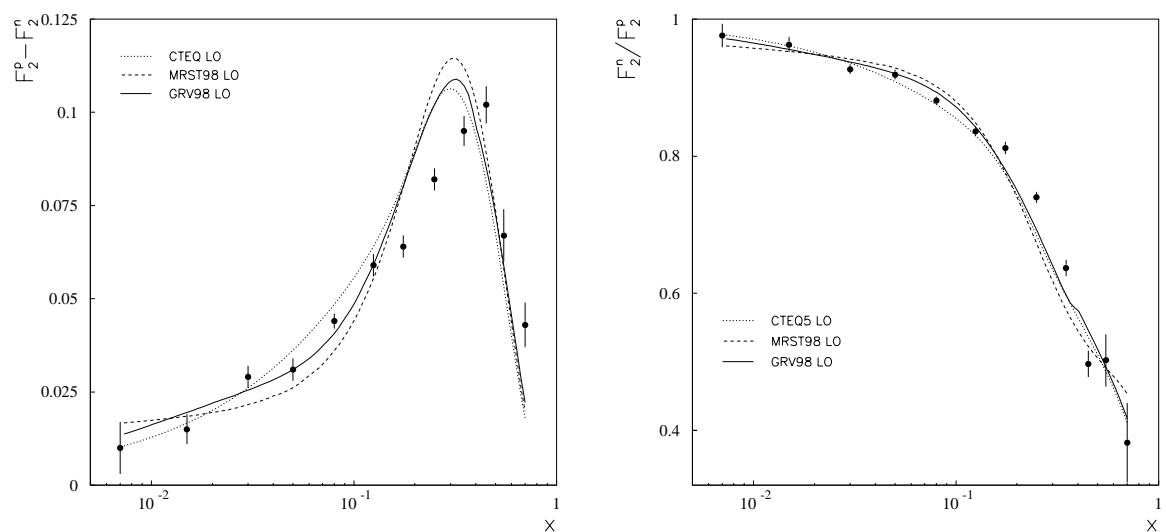

Figure 1: NMC data $F_{2}^{p}-F_{2}^{n}$ and $F_{2}^{n} / F_{2}^{p}$ against LO parameterizations.

in the deuteron, however, improve this situation, suggesting that indeed something is missing in the analysis of the deuteron structure function.

\section{Nuclear Effects}

Among the different models devised to account for nuclear effects in hard processes, available in the literature, in our analysis we choose to parameterize them with a slight variation of the usual pion model [10]. This choice is supported by the conceptual simplicity and phenomenological success of the pion model. Our aim for the time being is to emphasize the potential impact of these corrections in global analyses rather than an extremely precise extraction of parton densities, for which many other ingredients should also have to be taken into account.

The pion model proposes that a very small fraction of the deuteron momentum is carried by mesons, mainly pions, responsible for the nuclear binding. The consequent reduction of the light cone momentum fraction carried by nucleons produces in the structure function a depletion at large $x$ while the hard scattering over the pions enhance it at intermediate $x$. The model then implies for the deuteron structure function the self-evident convolution expression

$$
\begin{aligned}
2 F_{2}^{d} & \rightarrow \int_{x}^{2} d y\left[F_{2}^{p}(x / y)+F_{2}^{n}(x / y)\right] f_{p / d}(y) \\
& +3 \int_{x}^{2} d y F_{2}^{\pi}(x / y) f_{\pi / d}(y)
\end{aligned}
$$

so that, upon the introduction of the probability

$$
f_{p / d}(y)=\delta(1-y-\epsilon)
$$

one ends with

$$
\begin{aligned}
2 F_{2}^{d} & =\left[F_{2}^{p}\left(\frac{x}{1-\epsilon}\right)+F_{2}^{n}\left(\frac{x}{1-\epsilon}\right)\right] \theta(1-\epsilon-x) \\
& +3 \int_{x}^{2} d y F_{2}^{\pi}(x / y) f_{\pi / d}(y)
\end{aligned}
$$

For the pion structure function we use the parameterization of reference [12] without any free parameter, while the pion distribution inside the nucleus is written as [11]

$$
f_{\pi / d}(y) \propto\left(\frac{y}{c}\right)^{a}\left(1-\frac{y}{c}\right)^{b} ; f_{\pi / d}(y)=0 \text { if } y>c
$$

normalized in order to have

$$
\int_{0}^{2} d y \frac{y}{2} 3 f_{\pi / d}(y)=\epsilon
$$

being $\epsilon$ the fraction of the deuteron momentum carried by its pionic constituents.

In order to parameterize quark densities at the initial scale $Q_{0}^{2}$ we adopt the standard functional dependence as in [3], with an additional free parameter for the $\bar{d}-\bar{u}$ density.

Leaving aside the normalizations for valence quarks and gluons, which are fixed by charge and momentum conservation, the above distributions imply that 25 parameters, 22 corresponding to the parton densities plus the $\epsilon, a$, and $b$ parameters coming from the pion distribution in nuclei 
have to be adjusted. The parameter $c$ has been fixed to 1.11 in order to have $f_{\pi / d}(y)$ vanishingly small for $y>1$ as required by Drell Yan data.

The initial scale $Q_{0}^{2}$ is taken to be $1 \mathrm{GeV}^{2}$ and the evolution is performed using a variable number of active flavours with thresholds at $1.69 \mathrm{GeV}^{2}$ for charm, and $18.49 \mathrm{GeV}^{2}$ for bottom. Below these thresholds the corresponding contributions to the structure function are given by the related photon-gluon fusion diagrams. For the strong coupling $\alpha_{s}$ the LO expression with $\Lambda_{Q C D}=0.174 \mathrm{GeV}$ (for four flavours) is taken.

\section{Data}

The data set used in the global fitting procedure includes the following experiments:

- DIS $(\mu N \rightarrow \mu X)$

$$
\begin{aligned}
& \text { - NMC [7]: } F_{2}^{p}(130), F_{2}^{d}(130) \\
& \text { - BCDMS [13]: } F_{2}^{p}(177), F_{2}^{d}(159) \\
& \text { - E665 [14]: } F_{2}^{p}(53), F_{2}^{d}(53) \\
& \text { - H1 [15] (150), ZEUS [16] (158): } F_{2}^{p}
\end{aligned}
$$

- Drell-Yan $\left(p N \rightarrow \mu^{+} \mu^{-} X\right)$

$$
\text { - E866 [9]: } \sigma_{p d} / 2 \sigma_{p p}(11)
$$

- $W^{+} W^{-}$Asymmetry

$$
\text { - CDF [17]: }\left(\sigma_{\ell^{+}}-\sigma_{\ell^{-}}\right) /\left(\sigma_{\ell^{+}}+\sigma_{\ell^{-}}\right)(11)
$$

where we have excluded data with $x>0.75$, $x<0.001, Q^{2}<2 \mathrm{GeV}^{2}$, and $W^{2}<7 \mathrm{GeV}^{2}$ in order to avoid higher order QCD and mass effects. The quantities in brackets are the number of data points fitted for each observable.

\section{Results}

In Figure 2 we show the main features of the parton densities corresponding to two fits: the first one, labeled $\epsilon=0.03$, includes nuclear effects with pions carrying about $3 \%$ of the deuteron momentum. In the second, denoted as $\epsilon=0$, no nuclear effects have been taken into account.

In both fits, NMC data on $F_{2}^{p}-F_{2}^{n}$ have been included with an additional artificial weight in order to force the parton densities to reproduce the data. The main consequence of this is a modification of the $\bar{d}-\bar{u}$ density which spoils the agreement with E866 data. In Figure 2 we also include MRST LO parton densities for comparison, highlighting the fact that neither valence densities nor the sum of antiquark distributions are significantly changed. The inclusion of nuclear effects allows to conciliate the antagonism between E866 and NMC data. These effects, at least as they are modeled here, can not remove the discrepancy completely, but reduce slightly the total (unweighted) $\chi^{2}$.

Figure 3, and 4 show the degree of agreement between different sets of data and the outcome of the parton densities. Table 1 shows the $\chi^{2}$ values obtained for both sets. The third column shows the ones for the same set data using MRST LO parton densities. In the calculus of the total $\chi^{2}$ shown, we included the NMC difference observable, which was taken as an external constraint in the fit.

\begin{tabular}{|l|c|c|c|}
\hline & $\epsilon=0.03$ & $\epsilon=0$ & $M R S T 98$ \\
\hline$N M C F_{2}^{p}$ & 176 & 192 & 144 \\
$N M C F_{2}^{D}$ & 200 & 239 & 122 \\
$N M C F_{2}^{n}-F_{2}^{p}$ & 51 & 29 & 167 \\
\hline$B C D M S F_{2}^{p}$ & 289 & 295 & 299 \\
$B C D M S F_{2}^{D}$ & 207 & 272 & 263 \\
\hline$E 665 F_{2}^{p}$ & 59 & 62 & 62 \\
$E 665 F_{2}^{D}$ & 53 & 55 & 50 \\
\hline$H 1 F_{2}^{p}$ & 123 & 118 & 110 \\
\hline$Z E U S F_{2}^{p}$ & 259 & 238 & 245 \\
\hline$E 866 \sigma^{p D} / 2 \sigma^{p p}$ & 62 & 83 & 5 \\
\hline CDF Asymmetry & 18 & 13 & 21 \\
\hline$\chi^{2}$ (unweighted) & 1497 & 1596 & 1488 \\
\hline
\end{tabular}

Table 1: $\chi^{2}$ values obtained for different sets of data.

\section{Conclusions}

We have analyzed the incidence of nuclear effects in the deuteron structure function in the valence region finding that they help to moderate the apparent disagreement between NMC data on $F_{2}^{p}-F_{2}^{n}$ and current parton densities. The inclusion of this data in QCD fits with an artificial weight in order to force parton densities to reproduce it, requires sea quark densities which are not compatible with E866 data. This disagreement is somewhat moderated when nuclear effects are taken into account. The symmetry 

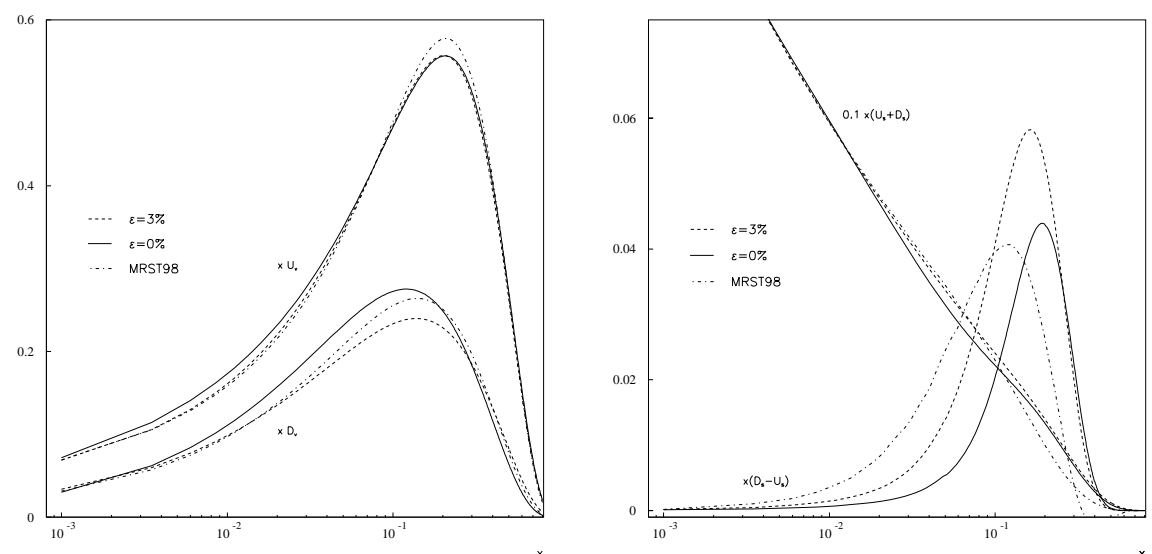

Figure 2: Parton densities extracted from the fits described in the text
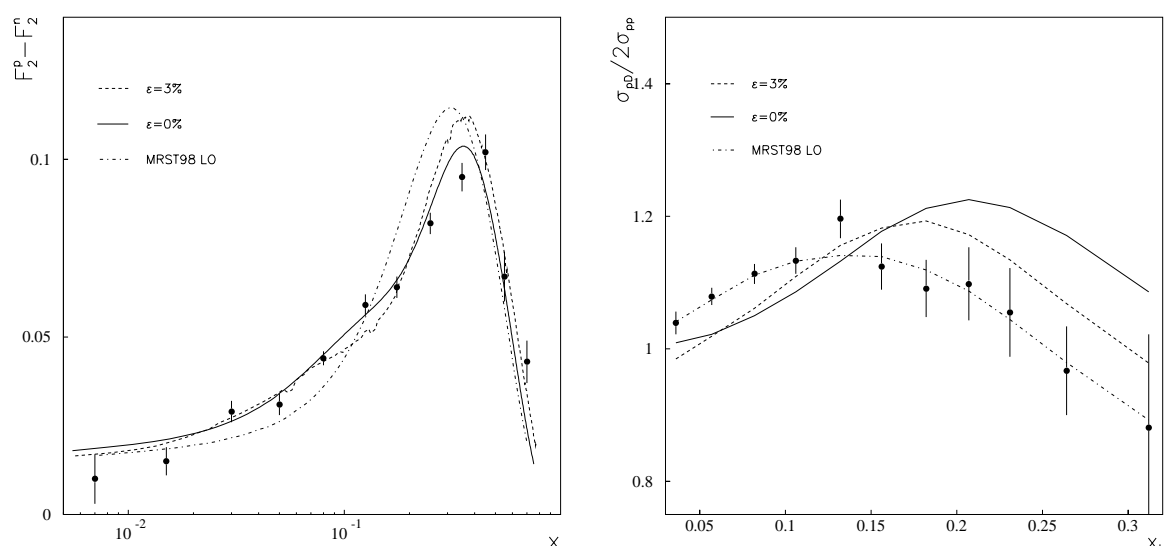

Figure 3: Global fit estimates for a) NMC data on $F_{2}^{p}-F_{2}^{n}$, and b) E866 data on $\sigma^{p D} / 2 \sigma^{p p}$

breaking between $\bar{u}$ and $\bar{d}$ quarks is found to be slightly larger than usual when including NMC data and it is slightly larger when nuclear effects are accounted for.

\section{Acknowledgments}

This research was supported in part by CONICET, ANPCyT and Fundación Antorchas Argentina

\section{References}

[1] C. A. García Canal, R. Sassot, Int. J. Mod. Phys. A (to be published).

[2] M. Glück, E. Reya, A. Vogt, Eur. Phys. J. C 5 (1998) 461.
[3] A. D. Martin, R. G. Roberts, W. J. Stirling, R. S. Thorne, Eur. Phys. J. C 4 (1998) 463.

[4] H. Lai. et al., Eur. Phys. J. C 12 (2000) 375.

[5] L. N. Epele et al., Phys. Lett. B 287 (1992) 247.

[6] S. Kumano, Phys. Rep. 303 (1998) 1.

[7] NMC Collaboration: M. Arneodo et al., Nucl. Phys. B 483 (1997) 3.

[8] A. Baldit et al., Phys. Lett. B 332 (1994) 244.

[9] E866 Collaboration: E. A. Hawker et al.,Phys. Rev. Lett. 80 (1998) 3715.

[10] E. L. Berger, F. Coester, R. B. Wiringa, Phys. Rev. D 29 (1984) 398.

[11] D. de Florian, et al., Z. Phys. B350 (1994) 55.

[12] P. J. Sutton et al., Phys. Rev. D 45 (1992) 2349.

[13] BCDMS Collaboration: A. C. Benvenuti et al., Phys. Lett. B 223 (1989) 485; Phys. Lett. B 237 (1989) 592. 

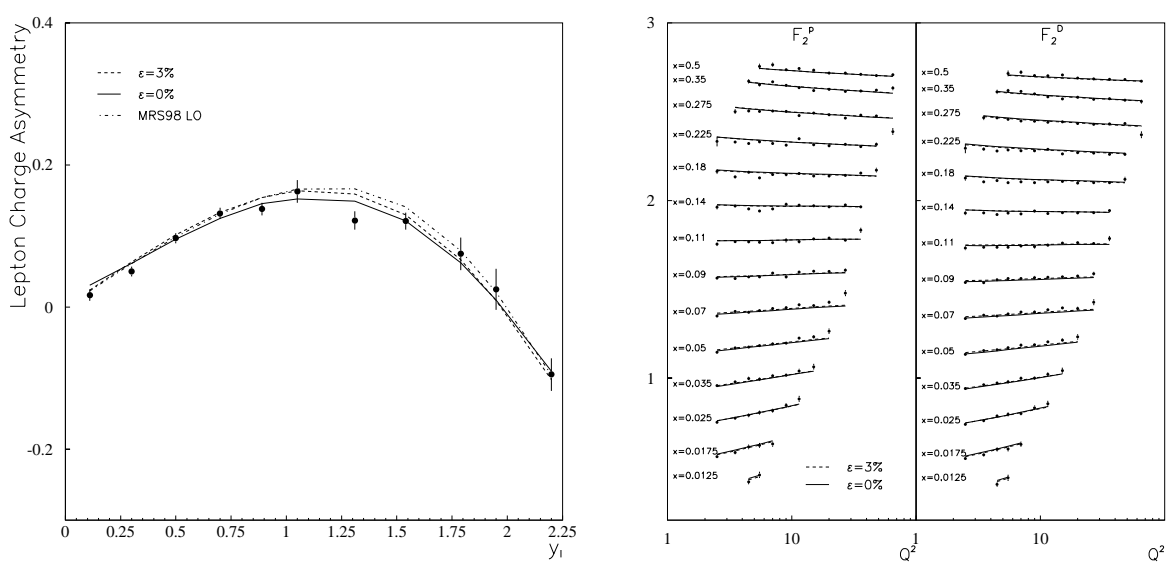

Figure 4: a) CDF W asymmetry data against the estimate from the fits described in the text; and b) Comparison between $F_{2}^{p}$ and $F_{2}^{D}$ as measured by NMC[7] and the above mentioned fits.

[14] E665 Collaboration: M. R. Adams et al., Phys. Rev. D 54 (1996) 3006.

[15] H1 Collaboration: S. Aid et al., Nucl. Phys. B 470 (1992) 3;

C. Adloff et al., Z. Physik C 72 (1996) 593.

[16] ZEUS Collaboration: Derrick et al., Z. Physik C 65 (1995) 379;

J. Breitweg et al., Phys. Lett. B 407 (1997) 402; Paper N-645 presented at International Europhysics Conference on High Energy Physics, HEP97, Jerusalem 1997.

[17] CDF Collaboration: F. Abe et al., Phys. Rev. Lett. 81 (1998) 5754. 